\title{
Suppression of Formation Flying Deviation in Near-Circular Orbits under the $J_{2}$ Perturbation
}

\author{
By Kenji Kitamura, ${ }^{1)}$ Katsuhiko YAmadA ${ }^{2}$ and Takeya SHIMA ${ }^{1)}$ \\ 1) Advanced Technology R\&D Center, Mitsubishi Electric Corporation, Amagasaki, Japan \\ 2) Graduate School of Engineering, Osaka University, Osaka, Japan
}

(Received July 31st, 2015)

\begin{abstract}
Station-keeping maneuvers are necessary for the precise formation flying of spacecraft, because various disturbing forces deviate their relative motion. The objective of this study is to derive a two-impulsive control law for the formation flying of two spacecraft in an orbit with a small eccentricity under the $J_{2}$ perturbation, especially one that suppresses the relative position deviation during the control period. Because of the $J_{2}$ perturbation, the relative position and velocity have secular terms. However, it is possible to compensate for the secular terms of the relative motion and create an artificial periodic motion by conducting the impulsive control. In this study, a state transition matrix of the relative motion based on the osculating orbital elements with the first order of $J_{2}$ is utilized, in order to calculate the secular and periodic terms of the relative motion, to derive an impulsive control law, and to determine the appropriate initial conditions for suppressing the deviation of formation flying. The numerical results of the proposed control law are compared with those of another method, which is based on the relative mean orbital elements, and it is shown that the proposed control law successfully suppresses the formation deviation.
\end{abstract}

Key Words: Formation Flying, $J_{2}$ Perturbation, State Transition Matrix, Impulsive Control

\section{Nomenclature}

$a \quad: \quad$ semi-major axis

$e:$ eccentricity

$i$ : inclination

$\omega:$ argument of perigee

$\Omega$ : right ascension of ascending node

$v$ : true anomaly

$\theta \quad: \quad$ argument of latitude

$n$ : mean angular motion

$J_{2} \quad$ : second order zonal harmonic coefficient of the Earth's gravitational potential

$r$ : position vector from the center of the Earth to the spacecraft

$r$ : distance from the center of the Earth to the spacecraft

$R_{e} \quad: \quad$ equatorial radius of the Earth

$t \quad:$ time

$T$ : orbital period

Subscripts

$0 \quad$ : initial states

$c$ : variables of the chief spacecraft

$d$ : variables of the deputy spacecraft

\section{Introduction}

The basic equations which describe the relative motion of two spacecraft in formation flying mode are the Hill-Clohessy-Wiltshire (HCW) equations ${ }^{1,2}$ ) and the Tshauner-Hempel (TH) equations., ${ }^{3,4)}$ These equations are derived under the assumptions that the Earth's gravitational field is perfectly spherical, and that the spacecraft are not subjected to any perturbing forces. However, the actual orbit of a spacecraft deviates from the nominal orbit because of various perturbing forces; thus, the relative motion of the spacecraft does not correspond to the solution obtained using the HCW or TH equations. 5) Among the various perturbing forces, one of the most influential perturbations is the $J_{2}$ perturbing force, which exists because of the Earth's oblateness. ${ }^{6}$ Yamada et al. $^{7,8)}$ derived the analytical state transition matrix (STM) of the TH equations under the effect of $J_{2}$. This STM incorporates the osculating orbital elements of the spacecraft, and enables the analytical treatment of relative motion.

Because of the $J_{2}$ perturbation, the relative motion of the spacecraft may deviate from the nominal motion, and it is necessary to conduct orbit maintenance control. D'Amico et al. derived the impulsive control law based on the relative mean eccentricity and inclination vectors. ${ }^{9-11)}$ Although the idea of relative eccentricity/inclination vector separation gives an immediate insight into the relative motion, and is useful for formulating the control law, it does not pay attention to the osculating relative motion, and may cause a relatively large formation deviation. In this study, the STM of Yamada et al. is used to calculate the osculating terms of the relative motion, to derive a two-impulsive control law and to determine the appropriate initial conditions for suppressing the formation deviation.

This paper is organized as follows. In section 2, the formation deviation based on the argument of latitude of the target spacecraft is formulated by means of the STM, 
incorporating the effect of $J_{2}$. In section 3, the necessary conditions for the impulsive control and the initial formation deviation are derived for the formation deviation to be periodic. Moreover, the remaining degrees of freedom of the initial formation deviation are utilized to suppress the formation deviation during the control period. In section 4 , numerical simulations are presented to show the validity of the proposed impulsive control law. Finally, section 5 presents our conclusions.

\section{Deviation of Relative Motion}

\subsection{Equation of relative motion under $\boldsymbol{J}_{\mathbf{2}}$}

In this paper, the target spacecraft flying in the reference orbit is named a chief, and the other spacecraft flying around the chief is named a deputy. In order to describe the relative motion of the two spacecraft, a local vertical/local horizontal (LVLH) coordinate system, centered at the chief is defined, whose $x$ axis points to the chief from the center of the Earth, $z$ axis is perpendicular to the orbital plane of the chief, and $y$ axis is chosen to complete the right hand coordinate system. The relative position of the deputy with respect to the chief is expressed in the LVLH coordinates as $(x, y, z)$, and the state vector of the relative motion is defined as follows:

$$
\boldsymbol{x}=\left[\begin{array}{llllll}
x & y & z & \dot{x} & \dot{y} & \dot{z}
\end{array}\right]^{T}
$$

The equations of the relative motion under the influence of $J_{2}$ are written as follows: ${ }^{8)}$

$$
\begin{aligned}
& {\left[\begin{array}{c}
\ddot{x}-2 w_{z} \dot{y}-\left(2 k+w_{z}^{2}\right) x-\dot{w}_{z} y+w_{x} w_{z} z \\
\ddot{y}+2 w_{z} \dot{x}-2 w_{x} \dot{z}+\dot{w}_{z} x+\left(k-w_{z}^{2}\right) y-\dot{w}_{x} z \\
\ddot{z}+2 w_{x} \dot{y}+w_{x} w_{z} x+\dot{w}_{x} y+k z
\end{array}\right]} \\
& =\boldsymbol{f}_{J}\left(\boldsymbol{r}_{\boldsymbol{d}}\right)-\boldsymbol{f}_{J}\left(\boldsymbol{r}_{\boldsymbol{c}}\right)
\end{aligned}
$$

where $w_{x}, w_{y}$, and $w_{z}$ are the components of the angular velocity vector of the LHVH frame with respect to the inertial coordinates, expressed in the LVLH. A scalar $k$ and a vector $\boldsymbol{f}_{J}(\boldsymbol{r})$ are defined as follows:

$$
\begin{gathered}
k=\mu / r_{c}^{3} \\
\boldsymbol{f}_{J}(\boldsymbol{r})=\frac{3 \mu J_{2} R^{2}}{2 r^{7}}\left[\left(5 r_{b}^{2}-r^{2}\right) \boldsymbol{r}-2 r^{2} r_{b}^{2} \hat{\boldsymbol{r}}_{b}\right]
\end{gathered}
$$

where $\hat{\boldsymbol{r}}_{b}$ is a unit vector pointing to the North Pole from the center of the Earth, and $r_{b}$ is defined as $r_{b}=\boldsymbol{r} \cdot \hat{\boldsymbol{r}}_{b}$.

\subsection{STM including the first order of $J_{2}$ and eccentricity}

Let us define the state vector at initial time $t_{0}$ as $\boldsymbol{x}_{0}$. Then, the state vector at time $t$ is expressed as follows:

$$
\boldsymbol{x}(t)=\Phi\left(t, t_{0}\right) \boldsymbol{x}_{0}
$$

where $\Phi\left(t, t_{0}\right)$ is an STM derived by Yamada et al. ${ }^{8)}$, which includes the first order of $J_{2}$. Let us assume that the osculating orbital elements of the chief at time $t_{0}$ are expressed as $a_{c 0}, e_{c 0}$, $i_{c 0}, \Omega_{c 0}, \omega_{c 0}, v_{c 0}, \theta_{c 0}$, and $M_{c 0}$, and that the nominal true anomaly and the nominal argument of latitude of the chief at time $t$ are expressed as $v_{c n}(t)$ and $\theta_{c n}(t)$, respectively, which are obtained by propagating $v_{c 0}$ and $\theta_{c 0}$ without any perturbations. The STM $\Phi\left(t, t_{0}\right)$ can be calculated by using these nominal orbital elements of the chief at time $t_{0}$ and $t$.

Assuming that $e_{c 0}$ is a small quantity of the first order, which is the same as $J_{2}$, the STM $\Phi\left(t, t_{0}\right)$ can be approximated in the first order as follows:

$$
\Phi\left(t, t_{0}\right)=\Phi^{C W}\left(t, t_{0}\right)+\delta \Phi^{e}\left(t, t_{0}\right)+\delta \Phi^{J}\left(t, t_{0}\right)
$$

where $\Phi^{C W}\left(t, t_{0}\right)$ corresponds to the STM of the HCW equations, which can be expressed as the summation of the periodic part $\Phi_{p}^{C W}(\phi)$ and the secular part $\Phi_{s}^{C W}(\tau)$ as follows:

$$
\Phi^{C W}\left(t, t_{0}\right)=\Phi_{p}^{C W}\left(\theta_{c n}(t)-\theta_{c 0}\right)+\Phi_{s}^{C W}\left(t-t_{0}\right)
$$

where $\Phi_{p}^{C W}(\phi)$ is a matrix given as follows:

$$
\Phi_{p}^{C W}=\left[\begin{array}{cccccc}
4-3 c_{\phi} & 0 & 0 & \frac{s_{\phi}}{n_{c 0}} & \frac{2\left(1-c_{\phi}\right)}{n_{c 0}} & 0 \\
6 s_{\phi} & 1 & 0 & \frac{2\left(c_{\phi}-1\right)}{n_{c 0}} & \frac{4 s_{\phi}}{n_{c 0}} & 0 \\
0 & 0 & c_{\phi} & 0 & 0 & \frac{s_{\phi}}{n_{c 0}} \\
3 n_{c 0} s_{\phi} & 0 & 0 & c_{\phi} & 2 s_{\phi} & 0 \\
6 n_{c 0}\left(c_{\phi}-1\right) & 0 & 0 & -2 s_{\phi} & 4 c_{\phi}-3 & 0 \\
0 & 0 & -n_{c 0} s_{\phi} & 0 & 0 & c_{\phi}
\end{array}\right]
$$

In Eq. (8), $s_{\phi}$ and $c_{\phi}$ represent $\sin \phi$ and $\cos \phi$, respectively. The nonzero components of $\Phi_{s}^{C W}(\tau)$ are only the $(2,1)$ and $(2,5)$ components, and these are given as follows:

$$
\Phi_{s}^{C W}(\tau)_{2,1}=-6 n_{c 0} \tau, \Phi_{s}^{C W}(\tau)_{2,5}=-3 \tau
$$

The components of $\delta \Phi^{e}\left(t, t_{0}\right)$ are given in Appendix A. Because the components of $\delta \Phi^{J}\left(t, t_{0}\right)$ are complicated, the details are omitted here.

\subsection{Deviation of the argument of latitude of the chief due to $J_{2}$}

Let us express the osculating argument of latitude of the chief spacecraft at time $t$ as $\theta_{c}(t)$. Because of the $J_{2}$ perturbation, $\theta_{c}(t)$ deviates from $\theta_{c n}(t)$, and is given by $\Delta \theta_{c}(t)=\theta_{c}(t)-\theta_{c n}(t) \cdot \Delta \theta_{c}(t)$ can be written in the first order of $J_{2}$ as follows ${ }^{8)}$ :

$$
\begin{aligned}
& \Delta \theta_{c}(t)= \\
& m_{c 0} \tau\left(4 \cos ^{2} i_{c 0}-1+3 \sin ^{2} i_{c 0} \cos 2 \theta_{c 0}\right) \\
& -(1 / 6) \gamma\left[28 \sin ^{2} i_{c 0} \cos ^{2} \theta_{c 0} \sin \left(\theta_{c n}(t)-\theta_{c 0}\right)\right. \\
& -24 \cos \theta_{c 0} \sin \theta_{c n}(t)+36 \cos ^{2} i_{c 0} \cos \theta_{c 0} \sin \theta_{c n}(t) \\
& +9 \cos \theta_{c 0} \sin \theta_{c 0}-15 \cos ^{2} i_{c 0} \cos \theta_{c 0} \sin \theta_{c 0} \\
& -28 \cos ^{2} i_{c 0} \sin \theta_{c 0} \cos \theta_{c n}(t)+16 \cos \theta_{c n}(t) \sin \theta_{c 0} \\
& \left.+7 \cos ^{2} i_{c 0} \sin \theta_{c n}(t) \cos \theta_{c n}(t)-\sin \theta_{c n}(t) \cos \theta_{c n}(t)\right]
\end{aligned}
$$

where $\gamma$ is defined as follows:

$$
\gamma=\frac{3}{2} J_{2}\left(\frac{R_{e}}{a}\right)^{2}
$$

\subsection{Formation deviation based on the argument of latitude of the chief}

Let us express the nominal formation at time $t$ as $\boldsymbol{x}_{n}(t)$. In this study, it is assumed that $\boldsymbol{x}_{n}(t)$ is determined based on the osculating argument of latitude of the chief $\theta_{c}(t)$, and that $\boldsymbol{x}_{n}(t)$ is explicitly written as a function of $\theta_{c}(t)$ as follows:

$$
\begin{aligned}
& \boldsymbol{x}_{n}(t)=\boldsymbol{x}_{n}\left(\theta_{c}(t)\right)
\end{aligned}
$$

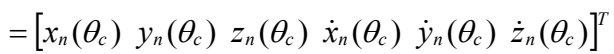

Furthermore, the following condition is considered between $x_{n}\left(\theta_{c}\right)$ and $\dot{y}_{n}\left(\theta_{c}\right)$ :

$$
2 n_{c 0} x_{n}\left(\theta_{c}\right)+\dot{y}_{n}\left(\theta_{c}\right)=0
$$

This is a necessary condition for cancelling the secular term in the solution of the HCW equations. Let us assume that the nominal formation is designed so that $\boldsymbol{x}_{n}\left(\theta_{c}\right)$ and $\boldsymbol{x}_{n}\left(\theta_{c 0}\right)$ 
have the following relationship.

$$
\boldsymbol{x}_{n}\left(\theta_{c}\right)=\Phi_{p}^{C W}\left(\theta_{c}-\theta_{c 0}\right) \boldsymbol{x}_{n}\left(\theta_{c 0}\right)
$$

Using the relationship $\theta_{c}(t)=\theta_{c n}(t)+\Delta \theta_{c}(t)$, the right hand side of Eq. (14) can be expanded as follows:

$$
\begin{aligned}
\boldsymbol{x}_{n}(t) & =\boldsymbol{x}_{n}\left(\theta_{c n}(t)+\Delta \theta_{c}(t)\right) \\
& =\Phi_{p}^{C W}\left(\theta_{c n}(t)+\Delta \theta_{c}(t)-\theta_{c 0}\right) \boldsymbol{x}_{n}\left(\theta_{c 0}\right) \\
& \approx\left[\Phi_{p}^{C W}\left(\theta_{c n}(t)-\theta_{c 0}\right)+\frac{\partial \Phi_{p}^{C W}}{\partial \phi} \Delta \theta_{c}(t)\right] \boldsymbol{x}_{n}\left(t_{0}\right)
\end{aligned}
$$

Then, the formation deviation based on the argument of latitude of the chief at time $t$ is written as follows:

$$
\begin{aligned}
\delta \boldsymbol{x}(t)= & \boldsymbol{x}(t)-\boldsymbol{x}_{n}(t) \\
= & \Phi\left(t, t_{0}\right) \boldsymbol{x}\left(t_{0}\right)-\boldsymbol{x}_{n}(t) \\
= & {\left[\Phi_{p}^{C W}\left(\theta_{c n}(t)-\theta_{c 0}\right)+\Phi_{s}^{C W}\left(t-t_{0}\right)\right.} \\
& \left.+\delta \Phi^{e}\left(t, t_{0}\right)+\delta \Phi^{J}\left(t, t_{0}\right)\right]\left[\boldsymbol{x}_{n}\left(t_{0}\right)+\delta \boldsymbol{x}\left(t_{0}\right)\right] \\
& -\left[\Phi_{p}^{C W}\left(\theta_{c n}(t)-\theta_{c 0}\right)+\frac{\partial \Phi_{p}^{C W}}{\partial \phi} \Delta \theta_{c}(t)\right] \boldsymbol{x}_{n}\left(t_{0}\right) \\
\approx & \Phi^{C W}\left(t, t_{0}\right) \delta \boldsymbol{x}\left(t_{0}\right)+\delta \Phi^{e}\left(t, t_{0}\right) \boldsymbol{x}_{n}\left(t_{0}\right) \\
+ & {\left[\delta \Phi^{J}\left(t, t_{0}\right)-\frac{\partial \Phi_{p}^{C W}}{\partial \phi} \Delta \theta_{c}(t)\right] \boldsymbol{x}_{n}\left(t_{0}\right) } \\
= & \delta \boldsymbol{x}^{C W}(t)+\delta \boldsymbol{x}^{e}(t)+\delta \boldsymbol{x}^{J}(t)
\end{aligned}
$$

where $\delta \boldsymbol{x}\left(t_{0}\right)$ is the formation deviation at time $t_{0}$. In the above equation, note that $\Phi_{s}^{C W}\left(t-t_{0}\right) \boldsymbol{x}_{n}\left(t_{0}\right)$ is $\mathbf{0}$ under the condition given in Eq. (13), and that $\delta \boldsymbol{x}^{C W}(t), \delta \boldsymbol{x}^{e}(t)$, and $\delta \boldsymbol{x}^{J}(t)$ are defined as follows:

$$
\begin{gathered}
\delta \boldsymbol{x}^{C W}(t)=\Phi^{C W}\left(t, t_{0}\right) \delta \boldsymbol{x}\left(t_{0}\right) \\
\delta \boldsymbol{x}^{e}(t)=\delta \Phi^{e}\left(t, t_{0}\right) \boldsymbol{x}_{n}\left(t_{0}\right) \\
\delta \boldsymbol{x}^{J}(t)=\left[\delta \Phi^{J}\left(t, t_{0}\right)-\frac{\partial \Phi_{p}^{C W}}{\partial \phi} \Delta \theta_{c}(t)\right] \boldsymbol{x}_{n}\left(t_{0}\right)
\end{gathered}
$$

where $\delta \boldsymbol{x}^{C W}(t)$ corresponds to the time propagation of initial formation deviation $\delta \boldsymbol{x}\left(t_{0}\right)$ by the STM of the HCW equations, and $\delta \boldsymbol{x}^{e}(t)$ and $\delta \boldsymbol{x}^{J}(t)$ are the formation deviations induced by the eccentricity and $J_{2}$, respectively. Let us define an angle $\psi$ as $\psi=\theta_{c}(t)-\theta_{c 0}$, and use this angle as an independent variable instead of $t$. Then, the components of Eq. (16) can be written as the functions of $\psi$ as follows:

$$
\begin{aligned}
\delta x(\psi)= & S_{3}^{x} \sin 3 \psi+C_{3}^{x}(\cos 3 \psi-1)+S_{2}^{x} \sin 2 \psi \\
& +C_{2}^{x}(\cos 2 \psi-1)+S_{1}^{x} \sin \psi+C_{1}^{x}(\cos \psi-1) \\
& +A \psi \sin \psi-B \psi \cos \psi+(4-3 \cos \psi) \delta x_{0} \\
& +\sin \psi \frac{\delta \dot{x}_{0}}{n_{c 0}}+2(1-\cos \psi) \frac{\delta \dot{y}_{0}}{n_{c 0}} \\
\delta y(\psi)= & S_{3}^{y} \sin 3 \psi+C_{3}^{y}(\cos 3 \psi-1)+S_{2}^{y} \sin 2 \psi \\
& +C_{2}^{y}(\cos 2 \psi-1)+S_{1}^{y} \sin \psi+C_{1}^{y}(\cos \psi-1) \\
& +2 B \psi \sin \psi+2 A \psi \cos \psi+F \psi \\
& -6(\psi-\sin \psi) \delta x_{0}+\delta y_{0}-2(1-\cos \psi) \frac{\delta x_{0}}{n_{c 0}} \\
& +(4 \sin \psi-3 \psi) \frac{\delta y_{0}}{n_{c 0}} \\
\delta z(\psi)= & S_{3}^{z} \sin 3 \psi+C_{3}^{z}(\cos 3 \psi-1)+S_{2}^{z} \sin 2 \psi \\
+ & C_{2}^{z}(\cos 2 \psi-1)+S_{1}^{z} \sin \psi+C_{1}^{z}(\cos \psi-1) \\
+ & D \psi \sin \psi-E \psi \cos \psi+\cos \psi \delta z_{0}+\sin \psi \frac{\delta \dot{z}_{0}}{n_{c 0}}
\end{aligned}
$$

$$
\begin{aligned}
\frac{\delta \dot{x}(\psi)}{n_{c 0}}= & S_{3}^{\dot{x}} \sin 3 \psi+C_{3}^{\dot{x}}(\cos 3 \psi-1)+S_{2}^{\dot{x}} \sin 2 \psi \\
& +C_{2}^{\dot{x}}(\cos 2 \psi-1)+S_{1}^{\dot{x}} \sin \psi+C_{1}^{\dot{x}}(\cos \psi-1) \\
& +B \psi \sin \psi+A \psi \cos \psi \\
& +3 \sin \psi \delta x_{0}+\cos \psi \frac{\delta \dot{x}_{0}}{n_{c 0}}+2 \sin \psi \frac{\delta \dot{y}_{0}}{n_{c 0}} \\
\frac{\delta \ddot{y}(\psi)}{n_{c 0}}= & S_{3}^{\dot{y}} \sin 3 \psi+C_{3}^{\dot{y}}(\cos 3 \psi-1)+S_{2}^{\dot{y}} \sin 2 \psi \\
& +C_{2}^{\dot{y}}(\cos 2 \psi-1)+S_{1}^{\dot{y}} \sin \psi+C_{1}^{\dot{y}}(\cos \psi-1) \\
& -2 A \psi \sin \psi+2 B \psi \cos \psi-6(1-\cos \psi) \delta x_{0} \\
& -2 \sin \psi \frac{\delta \dot{x}_{0}}{n_{c 0}}+(4 \cos \psi-3) \frac{\delta \dot{y}_{0}}{n_{c 0}} \\
\frac{\delta \dot{z}(\psi)}{n_{c 0}}= & S_{3}^{\dot{z}} \sin 3 \psi+C_{3}^{\dot{z}}(\cos 3 \psi-1)+S_{2}^{\dot{z}} \sin 2 \psi \\
& +C_{2}^{\dot{z}}(\cos 2 \psi-1)+S_{1}^{\dot{z}} \sin \psi+C_{1}^{\dot{z}}(\cos \psi-1) \\
& +E \psi \sin \psi+D \psi \cos \psi-\sin \psi \delta z_{0}+\cos \psi \frac{\delta \dot{z}_{0}}{n_{c 0}}
\end{aligned}
$$

where coefficients $A, B, C, D, E, F, S_{j}^{l}$, and $C_{j}^{l}$ are small quantities of the first order including $J_{2}$ or $e_{c 0}$, and have the unit of length. In the notation of $S_{j}^{l}$ and $C_{j}^{l}$, the subscript $j$ takes the values from 1 to 3 , and the superscript $l$ takes the values $x, y, z, \dot{x}, \dot{y}$, and $\dot{z}$. The details of these coefficients are given in Appendix B.

\section{Design of Impulsive Control}

\subsection{Formation deviation due to impulsive control}

In this section, the equations of the deviation of relative motion obtained in the previous section are utilized in order to derive the impulsive control law, and to maintain the formation flying.

Considering the structure of the matrix $\Phi^{C W}\left(t, t_{0}\right)$, Eq. (17) shows that the time propagation of the in-plane components of $\delta \boldsymbol{x}\left(t_{0}\right)$ and that of the out-of-plane components are independent. This means that the in-plane control and the out-of-plane control can be designed separately. In this study, let us make the following assumptions: the impulsive control is conducted once every $N$ orbital periods, the in-plane motion is maintained by two-impulsive controls $\Delta \boldsymbol{v}_{i}(i=1,2)$, the out-of-plane motion is maintained by one impulse $\Delta \boldsymbol{v}_{z}$, and the components of these impulsive controls are expressed as follows:

$$
\begin{gathered}
\Delta \boldsymbol{v}_{i}=\left[\begin{array}{lll}
\Delta v_{x i} & \Delta v_{y i} & 0
\end{array}\right]^{T} \quad(i=1,2) \\
\Delta \boldsymbol{v}_{z}=\left[\begin{array}{lll}
0 & 0 & \Delta v_{z}
\end{array}\right]^{T}
\end{gathered}
$$

In addition, let us assume that $\Delta \boldsymbol{v}_{1}$ and $\Delta \boldsymbol{v}_{2}$ are conducted when the arguments of latitude of the chief are $\theta_{c 0}+\phi_{1}$ and $\theta_{c 0}+\phi_{1}+\phi_{2}$, respectively, and that $\Delta \boldsymbol{v}_{z}$ is conducted at $\theta_{c 0}+\phi_{z}$, where $\phi_{i}$ and $\phi_{z}$ satisfy the conditions $0 \leq \phi_{i} \leq 2 \pi$ and $0 \leq \phi_{z} \leq 2 \pi$, respectively. These impulsive controls also induce the formation deviation $\delta \boldsymbol{x}^{V}(\psi)$.

Figure 1 shows the conceptual diagram of the position deviation due to the in-plane impulsive control. The first and second impulsive controls are conducted at $\psi=\phi_{1}$ and $\psi=\phi_{1}+\phi_{2}$, respectively. At these points, the gradient of the position deviation changes discontinuously. In Fig. 1, the 
dotted line shows the formation deviation due to the $J_{2}$ perturbation, and the solid line shows the total deviation after the impulsive control. The difference between these two lines corresponds to the formation deviation induced by the impulsive control, $\delta \boldsymbol{x}^{V}(\psi)$. The in-plane components of $\delta \boldsymbol{x}^{V}(\psi)$ can be written by means of the STM according to the range of $\psi$ as follows:

- If $0 \leq \psi<\phi_{1}$,

then there is no effect of $\Delta \boldsymbol{v}_{1}$ and $\Delta \boldsymbol{v}_{2}$ on $\delta \boldsymbol{x}^{V}(\psi)$. Therefore, the in-plane components of $\delta x^{V}(\psi)$ are all zeros, as given below.

$$
\delta x^{V}(\psi)=\delta y^{V}(\psi)=0, \quad \delta \dot{x}^{V}(\psi)=\delta y^{V}(\psi)=0
$$

- Else, if $\phi_{1} \leq \psi<\phi_{1}+\phi_{2}$

then the in-plane components of $\delta \boldsymbol{x}^{V}(\psi)$ can be calculated by propagating $\Delta \boldsymbol{v}_{1}$ using the STM as follows:

$$
\begin{aligned}
& {\left[\delta x^{V}(\psi) \delta y^{V}(\psi) \delta \dot{x}^{V}(\psi) \delta \dot{y}^{V}(\psi)\right]^{T}} \\
& =\Phi_{i n}^{C W}\left(t, t_{0}+\tau_{1}\right)\left[\begin{array}{llll}
0 & 0 & \Delta v_{x 1} & \Delta v_{y 1}
\end{array}\right]^{T}
\end{aligned}
$$

- Else, if $\phi_{1}+\phi_{2} \leq \psi \leq 2 N \pi$,

then the in-plane components of $\delta \boldsymbol{x}^{V}(\psi)$ can be obtained by propagating $\Delta \boldsymbol{v}_{1}$ and $\Delta \boldsymbol{v}_{2}$ using the STM as follows:

$$
\begin{aligned}
& {\left[\begin{array}{llll}
\delta x^{V}(\psi) & \delta y^{V}(\psi) & \delta \dot{x}^{V}(\psi) & \delta y^{V}(\psi)
\end{array}\right]^{T}} \\
& =\Phi_{i n}^{C W}\left(t, t_{0}+\tau_{1}+\tau_{2}\right)\left[\begin{array}{llll}
0 & 0 & \Delta v_{x 2} & \Delta v_{y 2}
\end{array}\right]^{T} \\
& +\Phi_{i n}^{C W}\left(t, t_{0}+\tau_{1}\right)\left[\begin{array}{llll}
0 & 0 & \Delta v_{x 1} & \Delta v_{y 1}
\end{array}\right]^{T}
\end{aligned}
$$

where $\Phi_{i n}^{C W}$ is a $4 \times 4$ matrix which consists of the in-plane components of $\Phi^{C W}$, and $\tau_{i}$ is set as $\tau_{i}=\phi_{i} / n_{c 0} \quad(i=1,2)$. Similarly, the out-of-plane components of $\delta \boldsymbol{x}^{V}(\psi)$ can be written according to the range of $\psi$ as follows:

- If $0 \leq \psi<\phi_{z}$,

then there is no effect of $\Delta \boldsymbol{v}_{z}$ on $\delta z^{V}(\psi)$. Therefore, the out-of-plane components of $\delta \boldsymbol{x}^{V}(\psi)$ are all zero as given below.

$$
\delta z^{V}(\psi)=0, \delta \dot{z}^{V}(\psi)=0
$$

- Else, if $\phi_{z} \leq \psi \leq 2 N \pi$,

then the out-of-plane components of $\delta \boldsymbol{x}^{V}(\psi)$ can be calculated by propagating $\Delta v_{z}$ using the STM as follows:

$$
\left[\begin{array}{l}
\delta z^{V}(\psi) \\
\delta \dot{z}^{V}(\psi)
\end{array}\right]=\Phi_{\text {out }}^{C W}\left(t, t_{0}+\tau_{z}\right)\left[\begin{array}{c}
0 \\
\Delta v_{z}
\end{array}\right]
$$

where $\Phi_{\text {out }}^{C W}$ is a $2 \times 2$ matrix that consists of the out-of-plane components of $\Phi^{C W}$, and $\tau_{z}$ is set as $\tau_{z}=\phi_{z} / n_{c 0}$. By taking the sum of Eq. (16) and $\delta \boldsymbol{x}^{V}(\psi)$, the formation deviation, including the effect of the impulsive controls as well as $J_{2}$ and $e_{c 0}$, is expressed as follows:

$$
\delta \boldsymbol{x}(\psi)=\delta \boldsymbol{x}^{C W}(\psi)+\delta \boldsymbol{x}^{e}(\psi)+\delta \boldsymbol{x}^{J}(\psi)+\delta \boldsymbol{x}^{V}(\psi)
$$

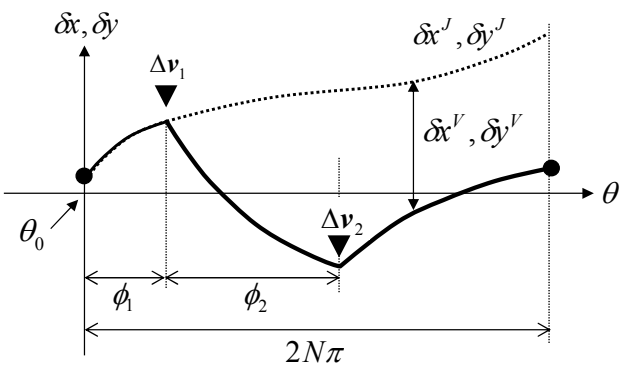

Fig. 1. Conceptual diagram of in-plane position deviation due to impulsive control.

\subsection{Conditions for periodicity of formation deviation}

As obvious from Eqs. (20)-(25), the formation deviation has secular terms, and increases with time. To compensate the secular terms and to create an artificial periodic motion, the impulsive control introduced in the previous section and the initial formation deviation $\delta \boldsymbol{x}_{0}$ need to be properly selected. Section 3.2 describes the necessary conditions for the impulsive control and initial formation deviation for creating the periodicity of the relative motion. In section 3.3, the remaining degrees of freedom of the initial formation deviation are utilized to suppress the formation deviation during the control period. Figure 2 shows the outline of the procedure described in sections 3.2 and 3.3. The total number of the variables is 14 , where 8 variables relate to the impulsive control $\left(\Delta v_{x 1}, \Delta v_{y 1}, \Delta v_{x 2}, \Delta v_{y 2}, \Delta v_{z}, \phi_{1}, \phi_{2}\right.$, and $\left.\phi_{z}\right)$ and 6 variables relate to the components of the initial formation deviation $\delta \boldsymbol{x}_{0}$. The total number of constraints is six, which corresponds to the periodic conditions of the six components of the formation deviation. As shown in step 1 in Fig. 2, the six variables related to the in-plane impulsive control $\left(\Delta v_{x 1}\right.$, $\Delta v_{y 1}, \Delta v_{x 2}, \Delta v_{y 2}, \phi_{1}$, and $\left.\phi_{2}\right)$ are determined from the periodic conditions of $\delta x, \delta \dot{x}$, and $\delta \dot{y}$, while the two variables related to the out-of-plane control $\left(\Delta v_{z}\right.$ and $\left.\phi_{z}\right)$ are determined from the conditions of $\delta z$ and $\delta \dot{z}$. To satisfy the periodic condition of $\delta y$, one degree of freedom of the initial formation deviation $\delta \boldsymbol{x}_{0}$ is used. In step 2 , the remaining five degrees of freedom of the initial formation deviation are utilized to minimize the square integral of the formation deviation during the control period.

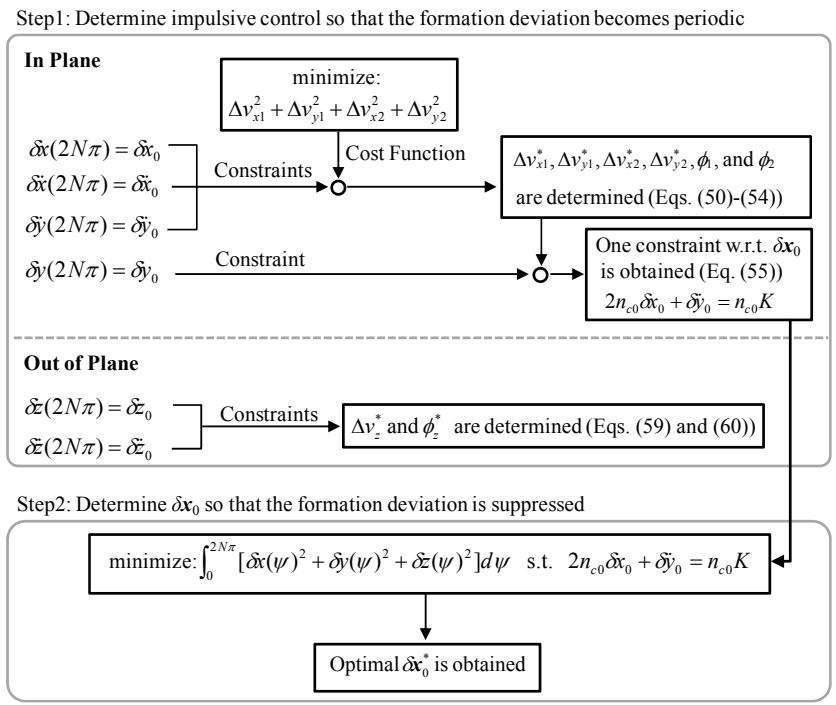

Fig. 2. Outline of the procedure to obtain impulsive control and optimal initial formation deviation described in sections 3.2 and 3.3.

The formation deviation after $N$ orbital periods is given by substituting $\psi=2 N \pi$ into Eq. (33) as given below.

$$
\begin{aligned}
\delta x(2 N \pi)= & \delta x_{0}-2 N \pi B-\frac{\sin \phi_{1}}{n_{c 0}} \Delta v_{x 1}-\frac{\sin \left(\phi_{1}+\phi_{2}\right)}{n_{c 0}} \Delta v_{x 2} \\
& +\frac{2\left(1-\cos \phi_{1}\right)}{n_{c 0}} \Delta v_{y 1}+\frac{2\left[1-\cos \left(\phi_{1}+\phi_{2}\right)\right]}{n_{c 0}} \Delta v_{y 2}
\end{aligned}
$$




$$
\begin{aligned}
\delta y(2 N \pi)= & \delta y_{0}-\frac{6 N \pi}{n_{c 0}}\left(2 n_{c 0} \delta x_{0}+\delta \ddot{y}_{0}\right)+2 N \pi(2 A+F) \\
& +\frac{2\left(\cos \phi_{1}-1\right)}{n_{c 0}} \Delta v_{x 1}+\frac{2\left[\cos \left(\phi_{1}+\phi_{2}\right)-1\right]}{n_{c 0}} \Delta v_{x 2} \\
& +\left(3 \phi_{1}-4 \sin \phi_{1}-6 N \pi\right) \frac{\Delta v_{y 2}}{n_{c 0}} \\
& +\left[3\left(\phi_{1}+\phi_{2}\right)-4 \sin \left(\phi_{1}+\phi_{2}\right)-6 N \pi\right] \frac{\Delta v_{y 2}}{n_{c 0}} \\
\delta z(2 N \pi)= & \delta z_{0}-2 N \pi E-\frac{\sin \phi_{1}}{n_{c 0}} \Delta v_{z} \\
\delta \dot{x}(2 N \pi)= & \delta \dot{x}_{0}+2 n_{c 0} N \pi A+\cos \phi_{1} \Delta v_{x 1}+\cos \left(\phi_{1}+\phi_{2}\right) \Delta v_{x 2} \\
& -2 \sin \phi_{1} \Delta v_{y 1}-2 \sin \left(\phi_{1}+\phi_{2}\right) \Delta v_{y 2} \\
\delta \dot{y}(2 N \pi)= & \delta \ddot{y}_{0}+4 n_{c 0} N \pi B \\
& +2 \sin \phi_{1} \Delta v_{x 1}+2 \sin \left(\phi_{1}+\phi_{2}\right) \Delta v_{x 2} \\
& +\left(4 \cos \phi_{1}-3\right) \Delta v_{y 1}+\left[4 \cos \left(\phi_{1}+\phi_{2}\right)-3\right] \Delta v_{y 2} \\
\delta \dot{z}(2 N \pi)= & \delta \dot{z}_{0}+2 n_{c 0} N \pi D+\cos \phi_{z} \Delta v_{z}
\end{aligned}
$$

For the formation deviation to be periodic, the following six equations need to be satisfied.

$$
\begin{aligned}
& \delta x(2 N \pi)=\delta x_{0} \\
& \delta y(2 N \pi)=\delta y_{0} \\
& \delta z(2 N \pi)=\delta z_{0} \\
& \delta \ddot{x}(2 N \pi)=\delta \dot{x}_{0} \\
& \delta \dot{y}(2 N \pi)=\delta \ddot{y}_{0} \\
& \delta \dot{z}(2 N \pi)=\delta \ddot{z}_{0}
\end{aligned}
$$

\subsubsection{Conditions for periodic in-plane motion}

Equations (40), (43), and (44) represent the necessary conditions for $\delta \dot{x}, \delta \dot{x}$, and $\delta \dot{y}$ to be periodic. By substituting these three equations into Eqs. (34), (37), and (38), the velocity increments $\Delta v_{x 1}, \Delta v_{y 1}$, and $\Delta v_{y 2}$ can be solved in terms of $\Delta v_{x 2}, \phi_{1}$, and $\phi_{2}$ as follows:

$$
\begin{aligned}
\Delta v_{x 1}=\Delta v_{x 2}+\frac{2 n_{c 0} N \pi}{\cos \phi_{1}-1}\left[A\left(\cos \phi_{1}-\cos \left(\phi_{1}+\phi_{2}\right)\right)\right. \\
\left.\quad+B\left(\sin \phi_{1}-\sin \left(\phi_{1}+\phi_{2}\right)\right)\right] \\
\Delta v_{y 1}=-\Delta v_{y 2} \quad \\
=\frac{\sin \phi_{2}}{2\left(\cos \phi_{1}-1\right)} \Delta v_{x 2}+\frac{n_{c 0} N \pi\left(B \cos \phi_{1}-A \sin \phi_{1}\right)}{\cos \phi_{1}-1}
\end{aligned}
$$

Because there still remain the degrees of freedom with respect to $\Delta v_{x 2}, \phi_{1}$, and $\phi_{2}$, let us determine these values, so that the following cost function $J_{v}$ is minimized.

$$
J_{v}=\Delta v_{x 1}^{2}+\Delta v_{x 2}^{2}+\Delta v_{y 1}^{2}+\Delta v_{y 2}^{2}
$$

The conditions to minimize $J_{v}$ are given as follows.

$$
\frac{\partial J_{v}}{\partial \Delta v_{x 2}}=0, \quad \frac{\partial J_{v}}{\partial \phi_{1}}=0, \quad \frac{\partial J_{v}}{\partial \phi_{2}}=0
$$

Then, $\Delta v_{x 2}, \phi_{1}$, and $\phi_{2}$ which satisfy the above equations are given as follows:

$$
\begin{gathered}
\cos \phi_{1}^{*}=\mp \frac{\Delta v_{x 2}^{*}=0}{\sqrt{A^{2}+B^{2}}}, \quad \sin \phi_{1}^{*}= \pm \frac{A}{\sqrt{A^{2}+B^{2}}} \\
\phi_{2}^{*}=\pi
\end{gathered}
$$

Therefore, $\Delta v_{x 1}, \Delta v_{y 1}$, and $\Delta v_{y 2}$ are determined as follows:

$$
\begin{gathered}
\Delta v_{x 1}^{*}=0 \\
\Delta v_{y 1}^{*}=-\Delta v_{y 2}^{*}= \pm \frac{1}{2} n_{c 0} N \pi \sqrt{A^{2}+B^{2}}
\end{gathered}
$$

In the above Eqs. (51) and (54), the double-signs correspond. Equation (41) represents the necessary conditions for $\delta y$ to be periodic. By substituting Eq. (41) into Eq. (35), the necessary condition for $\delta y$ is written as follows:

$$
2 n_{c 0} \delta x_{0}+\delta y_{0}=n_{c 0} K
$$

where $K$ is a small quantity of the first order with the dimension of length, and is expressed as follows.

$$
K=\frac{1}{3}(2 A+F)-\frac{\left(8 \sin \phi_{1}^{*}+3 \pi\right)}{6 N \pi} \frac{\Delta v_{y 1}^{*}}{n_{c 0}}
$$

\subsubsection{Conditions for periodic out-of-plane motion}

From the necessary conditions for $\delta z$ and $\delta \dot{z}$ to be periodic, the following equations are obtained.

$$
\begin{aligned}
& \sin \phi_{z} \Delta v_{z}=-2 n_{c 0} N \pi E \\
& \cos \phi_{z} \Delta v_{z}=-2 n_{c 0} N \pi D
\end{aligned}
$$

Therefore, $\phi_{z}$ and $\Delta v_{z}$ are determined as follows:

$$
\begin{gathered}
\phi_{z}^{*}=\operatorname{atan}(E / D) \\
\Delta v_{z}^{*}=-2 n_{c 0} N \pi\left(D \cos \phi_{z}^{*}+E \sin \phi_{z}^{*}\right)
\end{gathered}
$$

\subsection{Conditions for suppressing the position deviation}

From the results of the previous section, it can be seen that the amounts of each impulsive control and phase where the control should be executed are determined by Eqs. (50)-(54), (59), and (60), and that these values are unrelated to the initial formation deviation $\delta \boldsymbol{x}_{0}$. On the other hand, there is only one condition with respect to $\delta \boldsymbol{x}_{0}$, as given by Eq. (55), and $\delta \boldsymbol{x}_{0}$ cannot be uniquely determined from the necessary conditions for the periodic motion. This means that five degrees of freedom remain to determine $\delta \boldsymbol{x}_{0}$, and that it is possible to determine $\delta \boldsymbol{x}_{0}$, so that the position deviation during the $N$ orbital periods will be suppressed.

In order to suppress the position deviation, let us define the cost function $J(>0)$ by integrating the sum of squares of the position deviations for $N$ orbital periods as follows.

$$
J^{2} \equiv \frac{1}{2 N \pi} \int_{0}^{2 N \pi}\left[\delta x(\psi)^{2}+\delta y(\psi)^{2}+\delta z(\psi)^{2}\right] d \psi
$$

The necessary condition to minimize $J$ is given as follows.

$$
\frac{\partial J^{2}}{\partial \delta \boldsymbol{x}_{0}}=0
$$

Note that $\delta \boldsymbol{x}_{0}$ should also satisfy the equality constraint given by Eq. (55). Let us express the solution to this quadratic programming problem as $\delta \boldsymbol{x}_{0}^{*}$. The components of $\delta \boldsymbol{x}_{0}^{*}$ are given as follows.

$$
\begin{aligned}
\delta x_{0}^{*}= & \frac{1}{5 n_{c 0} N \pi}\left[5\left\{(4 N-1) \pi-2 \phi_{1}^{*}\right\} \cos \phi_{1}^{*}\right. \\
& \left.+2\left(3 \pi+11 \sin \phi_{1}\right)\right] \Delta v_{y 1}^{*} \\
& +\frac{1}{10}\left(10 N \pi B+44 K-2 C_{1}^{x}+4 S_{1}^{y}-8 F-3 A\right) \\
\delta y_{0}^{*}= & \frac{1}{20 n_{c 0} N \pi}\left[40\left\{(4 N-1) \pi-2 \phi_{1}^{*}\right\} \sin \phi_{1}^{*}\right. \\
& \left.\quad+3\left\{5 \pi\left((4 N-1) \pi-2 \phi_{1}^{*}\right)-16 \cos \phi_{1}^{*}\right\}\right] \Delta v_{y 1}^{*} \\
& +\frac{1}{5}\left(13 B+C_{1}^{y}-2 S_{1}^{x}\right)+C_{2}^{y}+C_{3}^{y} \\
& +(3 K-2 A-F) N \pi \\
\delta z_{0}^{*}= & \frac{1}{2 n N \pi}\left(2 N \pi-\phi_{z}\right) \sin \phi_{z} \Delta v_{z}+\frac{1}{2} D+\pi N E-C_{1}^{z}
\end{aligned}
$$




$$
\begin{aligned}
\delta \dot{x}_{0}^{*}= & \frac{1}{5 N \pi}\left[5\left\{(4 N-1) \pi-2 \phi_{1}^{*}\right\} \sin \phi_{1}^{*}-16 \cos \phi_{1}^{*}\right] \Delta v_{y 1}^{*} \\
& -\frac{1}{10} n_{c 0}\left(10 N \pi A+2 S_{1}^{x}+4 C_{1}^{y}-3 B\right) \\
\delta y_{0}^{*}= & -\frac{2}{5 N \pi}\left[5\left\{(4 N-1) \pi-2 \phi_{1}^{*}\right\} \cos \phi_{1}^{*}\right. \\
& \left.-2\left(3 \pi+11 \sin \phi_{1}^{*}\right)\right] \Delta v_{y 1}^{*} \\
& -\frac{1}{5} n_{c 0}\left(-10 N \pi B-39 K-4 S_{1}^{y}+2 C_{1}^{x}+8 F+3 A\right) \\
\delta z_{0}^{*}= & \left.\left.-\frac{1}{5 N \pi}\left[\left(2 N \pi-\phi_{z}^{*}\right)\right\} \cos \phi_{z}^{*}+\sin \phi_{z}^{*}\right)\right] \Delta v_{z}^{*} \\
& -\frac{1}{2} n_{c 0}\left(E+2 N \pi D+2 S_{1}^{z}\right)
\end{aligned}
$$

\subsection{Control to follow the desired periodic motion}

In order to realize the desired periodic motion derived in the previous section, the formation deviation must coincide with $\delta \boldsymbol{x}_{0}^{*}$ at the time when the argument of latitude of the chief is $\theta_{c 0}$. In this section, it is assumed that $\delta \boldsymbol{x}_{0}$ has a minor deviation with respect to $\delta \boldsymbol{x}_{0}^{*}$ at $\theta_{c}=\theta_{c 0}$, and the differential correction of the impulsive controls is utilized to follow the desired periodic motion.

From Eq. (33), the formation deviation at $\theta_{c}=\theta_{c 0}+2 N \pi$ is expressed as a function of $\delta \boldsymbol{x}_{0}, \Delta v_{y 1}, \Delta v_{y 2}, \Delta v_{z}, \phi_{1}, \phi_{2}$, and $\phi_{z}$ as follows:

$$
\delta \boldsymbol{x}(2 N \pi)=\boldsymbol{F}\left(\delta \boldsymbol{x}_{0}, \boldsymbol{X}\right)
$$

where the vector $\boldsymbol{X}$ is defined as follows:

$$
\boldsymbol{X}=\left[\begin{array}{llllll}
\Delta v_{y 1} & \Delta v_{y 2} & \Delta v_{z} & \phi_{1} & \phi_{2} & \phi_{z}
\end{array}\right]^{T}
$$

The periodic conditions satisfy the following equation.

$$
\delta \boldsymbol{x}_{0}^{*}=\boldsymbol{F}\left(\delta \boldsymbol{x}_{0}^{*}, \boldsymbol{X}^{*}\right)
$$

where the components of $\boldsymbol{X}^{*}$ are given in section 3.2. Let us assume that $\delta \boldsymbol{x}_{0}$ has a minor deviation with respect to $\delta \boldsymbol{x}_{0}^{*}$, which can be expressed as $\delta \boldsymbol{x}_{0}=\delta \boldsymbol{x}_{0}^{*}+D \delta \boldsymbol{x}_{0}$. For $\delta \boldsymbol{x}(2 N \pi)$ to coincide with $\delta \boldsymbol{x}_{0}^{*}$ after $N$ orbital periods, the impulsive controls will have to be corrected from those derived in section 3.2. Let us express this correction as $D \boldsymbol{X}=\left[\begin{array}{llllll}D \Delta v_{y 1} & D \Delta v_{y 2} & D \Delta v_{z} & D \phi_{1} & D \phi_{2} & D \phi_{z}\end{array}\right]^{T}$; then, the following equation will be satisfied.

$$
\delta \boldsymbol{x}_{0}^{*}=\boldsymbol{F}\left(\delta \boldsymbol{x}_{0}^{*}+D \delta \boldsymbol{x}_{0}, \boldsymbol{X}^{*}+D \boldsymbol{X}\right)
$$

By expanding the right hand side of Eq. (72) with respect to $\delta \boldsymbol{x}_{0}$ and $\boldsymbol{X}$, the following equation is obtained.

$$
\boldsymbol{0}_{6 \times 1}=\left[\frac{\partial \boldsymbol{F}}{\partial \delta \boldsymbol{x}_{0}}\right]_{*} D \delta \boldsymbol{x}_{0}+\left[\frac{\partial \boldsymbol{F}}{\partial \boldsymbol{X}}\right]_{*} D \boldsymbol{X}
$$

From Eq. (17), $\left[\partial \boldsymbol{F} / \partial \boldsymbol{x}_{0}\right]_{*}$ can be calculated by evaluating the SMT $\Phi^{C W}\left(t, t_{0}\right)$ for $N$ orbital periods, and is given as follows.

$$
\left[\frac{\partial \boldsymbol{F}}{\partial \delta \boldsymbol{x}_{0}}\right]_{*}=\left[\begin{array}{cccccc}
1 & 0 & 0 & 0 & 0 & 0 \\
-12 N \pi & 1 & 0 & 0 & -6 N \pi / n_{c 0} & 0 \\
0 & 0 & 1 & 0 & 0 & 0 \\
0 & 0 & 0 & 1 & 0 & 0 \\
0 & 0 & 0 & 0 & 1 & 0 \\
0 & 0 & 0 & 0 & 0 & 1
\end{array}\right]
$$

Here, $[\partial \boldsymbol{F} / \partial \boldsymbol{X}]_{*}$ is a Jacobin matrix of $\delta \boldsymbol{x}^{V}(\psi)$ with respect to $\boldsymbol{X}$, evaluated at $\psi=2 N \pi$, and is given as follows:

$$
\left[\frac{\partial \boldsymbol{F}}{\partial \boldsymbol{X}}\right]_{*}=\left[\begin{array}{ll}
G_{v} & G_{\phi}
\end{array}\right]
$$

$$
\begin{gathered}
G_{v}=\left[\begin{array}{ccc}
\frac{2\left(1-c_{\phi 1}^{*}\right)}{n_{c 0}} & \frac{2\left(1+c_{\phi 1}^{*}\right)}{n_{c 0}} & 0 \\
\frac{\kappa-4 s_{\phi 1}^{*}}{n_{c 0}} & \frac{\kappa+3 \pi+4 s_{\phi 1}^{*}}{n_{c 0}} & 0 \\
0 & 0 & -\frac{s_{\phi z}^{*}}{n_{c 0}} \\
-2 s_{\phi 1}^{*} & 2 s_{\phi 1}^{*} & 0 \\
-3+4 c_{\phi 1}^{*} & -3-4 c_{\phi 1}^{*} & 0 \\
0 & 0 & c_{\phi z}^{*}
\end{array}\right] \\
G_{\phi}=\left[\begin{array}{ccc}
\frac{4 s_{\phi 1}^{*} \Delta v_{y 1}^{*}}{n_{c 0}} & \frac{2 s_{\phi 1}^{*} \Delta v_{y 1}^{*}}{n_{c 0}} & 0 \\
-\frac{8 c_{\phi 1}^{*} \Delta v_{y 1}^{*}}{n_{c 0}}-\frac{\left(3+4 c_{\phi 1}^{*}\right) \Delta v_{y 1}^{*}}{n_{c 0}} & 0 \\
0 & 0 & -\frac{c_{\phi z}^{*} \Delta v_{z}^{*}}{n_{c 0}} \\
-4 c_{\phi 1}^{*} \Delta v_{y 1}^{*} & -2 c_{\phi 1}^{*} \Delta v_{y 1}^{*} & 0 \\
-8 s_{\phi 1}^{*} \Delta v_{y 1}^{*} & -4 s_{\phi 1}^{*} \Delta v_{y 1}^{*} & 0 \\
0 & 0 & -s_{\phi x}^{*} \Delta v_{z}^{*}
\end{array}\right]
\end{gathered}
$$

where $s_{\phi 1}^{*}, c_{\phi 1}^{*}, s_{\phi k}^{*}, s_{\phi k}^{*}$, and $\kappa$ are set as $s_{\phi 1}^{*}=\sin \phi_{1}^{*}$, $c_{\phi 1}^{*}=\cos \phi_{1}^{*}, \quad s_{\phi z}^{*}=\sin \phi_{z}^{*}, \quad c_{\phi z}^{*}=\cos \phi_{z}^{*}$, and $\kappa=3\left(\phi_{1}^{*}-2 N \pi\right)$, respectively. From Eq. (73), the correction of the impulsive controls $D \boldsymbol{X}$ is given as follows:

$$
D \boldsymbol{X}=-\left[\frac{\partial \boldsymbol{F}}{\partial \boldsymbol{X}}\right]_{*}^{-1}\left[\frac{\partial \boldsymbol{F}}{\partial \delta \boldsymbol{x}_{0}}\right]_{*} D \delta \boldsymbol{x}_{0}
$$

\subsection{Bias removal by $P I$ control}

If the formation deviation at $\theta_{c}=\theta_{c 0}$ coincides with $\delta x_{0}^{*}$, it can be expected that the norms of $\delta x, \delta y$, and $\delta z$ are suppressed, and that the averages of these variables become almost zero during the control period. However, the STM used in this study considers only the first order of $J_{2}$ and eccentricity, and some errors still remain. Therefore, it is difficult to make the averages of the position deviation exactly zero by utilizing the analytically derived $\delta x_{0}^{*}$. Because $\delta y$ has a secular term increasing with time, $|\delta y|$ becomes large when compared with $|\delta x|$ and $|\delta z|$, and removing the bias of dy contributes to suppressing the whole position deviation. In order to remove the bias of $\delta y$, let us modify $\delta y_{0}^{*}$, as will be done by a PI controller, as follows:

$$
\delta y_{0}^{* *}=\delta y_{0}^{*}-k_{P} \delta y_{\text {mean }}-k_{I} \sum \delta y_{\text {mean }}
$$

where $\delta y_{\text {mean }}$ is the mean of the upper peak $\delta y_{p}^{+}$and the lower peak $\delta y_{p}^{-}$of $\delta y$ during the $N$ orbital periods, and is expressed as follows:

$$
\delta y_{\text {mean }}=\left(\delta y_{p}^{+}+\delta y_{p}^{-}\right) / 2
$$

In Eq. (79), $k_{p}$ and $k_{I}$ represent the proportional and integral gain, respectively. By using $\delta y_{0}^{* *}$ instead of $\delta y_{0}^{*}$ in the differential correction method described in section 3.4, it can be expected that $\delta y_{\text {mean }}$ will become close to zero with time.

\section{Numerical Examples}

In order to evaluate the validity of the proposed impulsive control law, some numerical examples are shown in this section. Here, the nominal formation flying is assumed as a helix formation, defined by the following equations.

$$
x_{n}\left(\theta_{c}(t)\right)=R_{x z} \sin \left(\theta_{c}(t)-\theta_{c 0}+\alpha\right)
$$




$$
\begin{aligned}
& y_{n}\left(\theta_{c}(t)\right)=2 R_{x z}\left[\cos \left(\theta_{c}(t)-\theta_{c 0}+\alpha\right)-\cos \alpha\right] \\
& z_{n}\left(\theta_{c}(t)\right)=R_{x z} \cos \left(\theta_{c}(t)-\theta_{c 0}+\alpha\right) \\
& \dot{x}_{n}\left(\theta_{c}(t)\right)=n_{c 0} R_{x z} \cos \left(\theta_{c}(t)-\theta_{c 0}+\alpha\right) \\
& \dot{y}_{n}\left(\theta_{c}(t)\right)=-2 n_{c 0} R_{x z} \sin \left(\theta_{c}(t)-\theta_{c 0}+\alpha\right) \\
& \dot{z}_{n}\left(\theta_{c}(t)\right)=-n_{c 0} R_{x z} \sin \left(\theta_{c}(t)-\theta_{c 0}+\alpha\right)
\end{aligned}
$$

Figure 3 shows the trajectory of the helix formation flying in the Hill coordinates system. In this helix formation, the locus of the relative motion is a circle of radius $R_{x z}$ and initial phase angle $\alpha$. This formation has the advantage of a lower collision risk. From the results of section 3.2, $\Delta v_{y 1}^{*}, \Delta v_{z}^{*}, \phi_{1}^{*}$, and $\phi_{z}^{*}$ are given as follows:

$$
\begin{gathered}
\Delta v_{y 1}^{*}= \pm \frac{1}{4} R_{x z} N \gamma m_{c 0} \pi\left|1-5 \cos ^{2} i\right| \\
\Delta v_{z}^{*}=2 R_{x z} N \gamma m_{c 0} \pi \sin ^{2} i_{c 0} \sin \left(\alpha-\theta_{c 0}\right) \sin \left(\theta_{c 0}+\phi_{z}^{*}\right) \\
\cos \phi_{1}^{*}=\mp \cos \alpha, \sin \phi_{1}^{*}= \pm \sin \alpha \\
\tan \phi_{z}^{*}=\cot \left(\theta_{c 0}\right)
\end{gathered}
$$

where the double-signs correspond. Eq. (87) shows that $\Delta v_{y 1}^{*}$ is unrelated to $\theta_{c 0}$ and $\alpha$, and that it is dependent on the size of the helix $R_{x z}$. From Eq. (88), it can be seen that $\Delta v_{z}^{*}$ is zero if $\theta_{c 0}=\alpha$. It should also be noted that the absolute value of the impulsive control per orbital period is unrelated to $N$, as given in the following equation.

$$
\begin{aligned}
& \frac{\left|\Delta v_{y 1}^{*}\right|+\left|\Delta v_{y 2}^{*}\right|+\left|\Delta v_{z}^{*}\right|}{N} \\
& =(1 / 2) R_{x z} m m_{c 0} \pi\left(\left|1-5 \cos ^{2} i\right|+4 \sin ^{2} i_{c 0}\left|\sin \left(\alpha-\theta_{c 0}\right)\right|\right)
\end{aligned}
$$

With regard to the initial conditions of the chief and the simulation parameters, let us assume two cases as shown in Table 1 .

For each case, two types of impulsive control laws are examined: one is the law proposed in section 3 (Control-A), and the other is the law utilizing the relative mean orbital elements $^{3,4)}$ (Control-B). In control-B, the relative in-plane and out-of-plane motions are described by the relative mean eccentricity and inclination vector, respectively, as the general station-keeping controls of a geostationary spacecraft. Let us call the four simulation cases, case 1-A, case 1-B, case 2-A, and case 2-B. In all the cases, the initial value of $\delta \boldsymbol{x}_{0}$ is set at $\delta \boldsymbol{x}_{0}^{*}$, as given by Eqs. (63)-(68).

Table 1. Initial Conditions of the chief, and the simulation parameters.

\begin{tabular}{|c|c|c|c|}
\hline elements & unit & case 1 & case 2 \\
\hline \hline$a_{c 0}$ & {$[\mathrm{~km}]$} & $R_{e}+638.5$ & $R_{e}+635.0$ \\
\hline$e_{c 0}$ & {$[-]$} & 0.0015 & 0.0010 \\
\hline$i_{c 0}$ & {$[\mathrm{deg}]$} & 80.0 & 97.9 \\
\hline$\Omega_{c 0}$ & {$[\mathrm{deg}]$} & 10.0 & 0.0 \\
\hline$\omega_{c 0}$ & {$[\mathrm{deg}]$} & 20.0 & 0.0 \\
\hline$v_{c 0}$ & {$[\mathrm{deg}]$} & 10.0 & 130.0 \\
\hline$\theta_{c 0}$ & {$[\mathrm{deg}]$} & 30.0 & 130.0 \\
\hline$R_{x z}$ & {$[\mathrm{~km}]$} & 1.0 & 3.0 \\
\hline$\alpha$ & {$[\mathrm{deg}]$} & 30.5 & 40.0 \\
\hline$k_{P}$ & {$[-]$} & 0.3 & 0.3 \\
\hline$k_{I}$ & {$[-]$} & 0.5 & 0.5 \\
\hline
\end{tabular}

The results of the time history of the position deviation and the impulsive control are shown in Figs. 4 and 5. The control period $N$ is set as $N=2$ and the total simulation time is 12 orbital periods for each case. Although there are some transients at the beginning in cases 1-B and 2-B, the norms of the impulsive control are almost the same for both types of control laws. However, with regard to the position deviation, the deviations in cases 1-A and 2-A are smaller than those in cases 1-B and 2-B. This shows that the proposed control law can suppress the formation deviation successfully.

Figures 6 and 7 show the values of the cost function $J$, defined by Eq. (61), when the control cycle $N$ is changed from 1 to 12 . In the figures, the blue solid line and the blue dashed line show the analytical and simulation results, respectively, of the proposed control-A law. The red dashed line shows the results of control-B, where the relative mean orbital elements are used. For comparison, the results of control-B are also shown with the initial formation deviation set at zero (red dotted line).

As long as the control period $N$ is small, the results of the analysis and simulation of control-A almost agree, and they are small when compared to the results of control-B. Comparing the red dotted line with the red dashed line, it can be seen that as long as the control period $N$ is large, control-B results in smaller formation deviation with the initial formation deviation set at zero, than with the initial formation deviation set as Eqs. (63)-(68). Therefore, it is not always true that the proposed initial formation deviation can suppress the formation deviation if control-B is conducted. This is because the proposed initial formation deviation is determined based on the osculating orbital elements, while control-B is conducted based on the relative mean orbital elements.

If $N$ becomes larger, the difference between the analysis and simulation results of control-A increases. The reason for this difference can be explained as follows. The STM in Eq. (5) originally incorporates only the first order of $J_{2}$. Although the STM can predict the relative motion in a relatively short time range, it is not suitable for a long time prediction. Therefore, if $N$ is large, the prediction error makes the cost function of the simulation results of control-A larger than that of the analytical results of control-A.

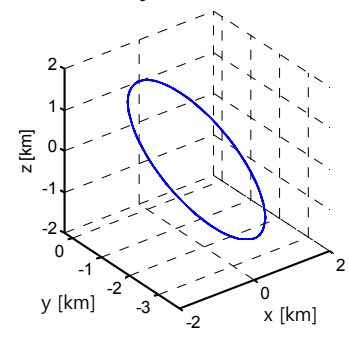

(a) $3 \mathrm{D}$ view

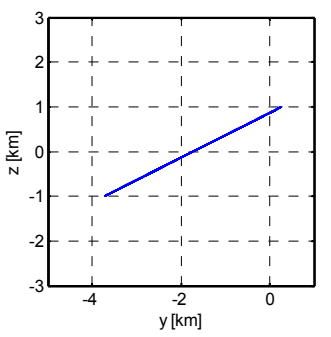

(c) y-z view

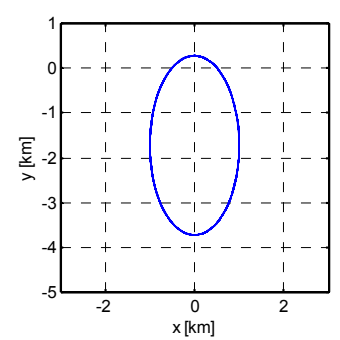

(b) $\mathrm{x}-\mathrm{y}$ view

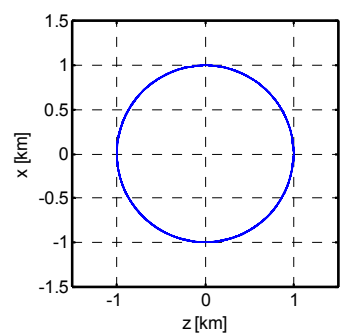

(d) z-x view
Fig. 3. Trajectory of the nominal helix formation flying in the Hill coordinate system (case-1). 


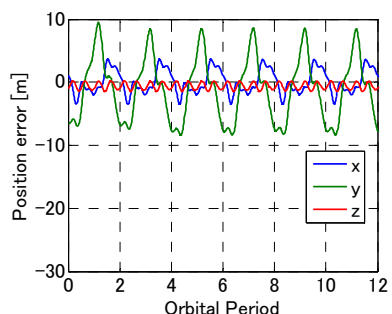

(a) Position deviation (case 1-A)

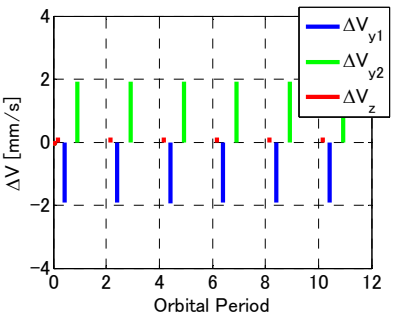

(c) Control Input (case 1-A)

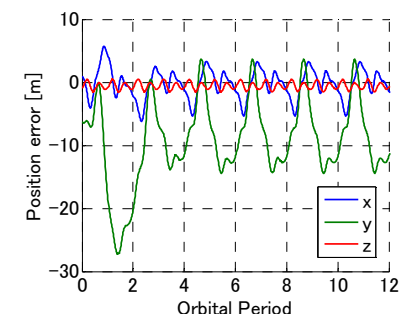

(b) Position deviation (case 1-B)

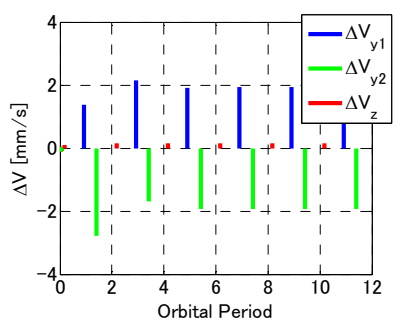

(d) Control Input (case 1-B)
Fig. 4. Time history of the position deviation and the impulsive control (cases 1-A and 1-B).

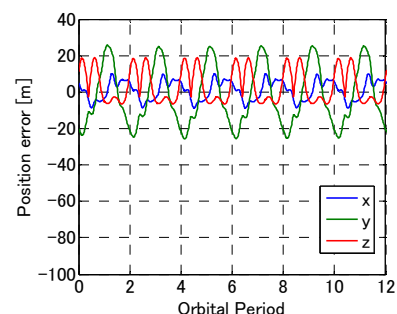

(a) Position deviation (case 2-A)

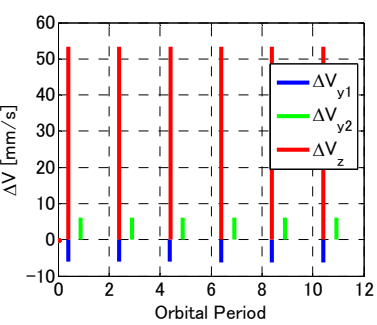

(c) Control Input (case 2-A)

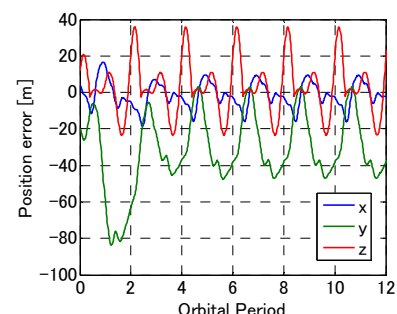

(b) Position deviation (case 2-B)

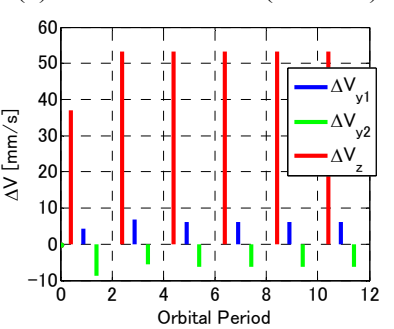

(d) Control Input (case 2-B)
Fig. 5. Time history of the position deviation and the impulsive control (cases 2-A and 2-B).

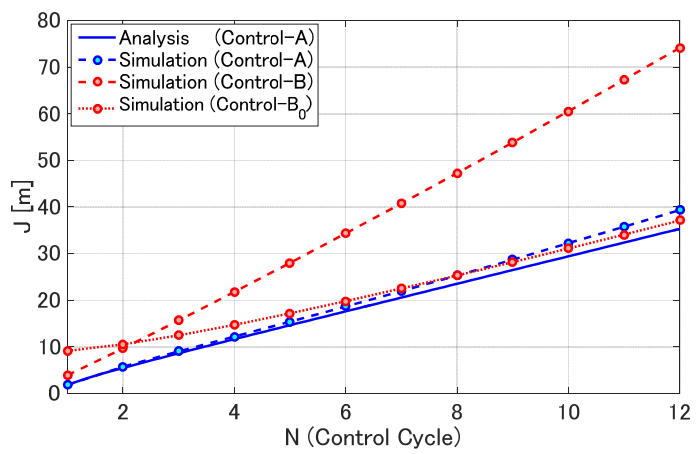

Fig. 6. Comparison of cost function $J$ (cases 1-A and 1-B).

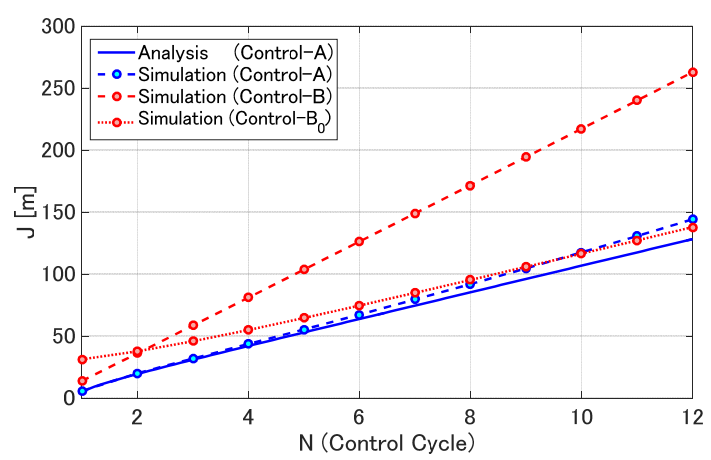

Fig. 7. Comparison of cost function $J$ (cases 2-A and 2-B).

\section{Conclusions}

In this study, an impulsive control law is derived for suppressing the position deviation of formation flying of two spacecraft in a near circular orbit under the $J_{2}$ perturbation. In the proposed control law, the in-plane relative motion is maintained by two-impulsive controls, and the out-of-plane motion by a single impulse. The conditions for the periodicity of the relative motion are analytically derived, and the appropriate initial values of the formation deviation, which suppresses the relative position deviation during a control period, are determined. Numerical simulation is conducted in order to validate the analytical solution, and to compare the proposed law with another law, which utilizes the relative mean orbital elements. The numerical simulations show that if the control period is relatively small (up to four or five orbital periods), the proposed impulsive control law can effectively suppress the formation deviation without using additional velocity increments.

\section{Appendix A. Components of $\delta \Phi^{e}\left(t, t_{0}\right)$}

Setting the $(i, j)$ components of $\delta \Phi^{e}\left(t, t_{0}\right)$ as $\delta \Phi_{i j}^{e}$, the nonzero components are expressed as follows:

$$
\begin{aligned}
& \delta \Phi_{11}^{e}=-2 e_{c 0}\left[\cos \left(v_{c n}(t)-2 v_{c 0}\right)-5 \cos v_{c 0}+4 \cos v_{c n}(t)\right. \\
&-\left.-3 n_{c 0} \tau \sin v_{c n}(t)\right] \\
& \delta \Phi_{12}^{e}=-e_{c 0}\left[\sin \left(v_{c n}(t)-2 v_{c 0}\right)-\sin v_{c n}(t)+2 \sin v_{c 0}\right]\left.+6 n_{c 0} \tau \sin v_{c n}(t)\right] \\
& \delta \Phi_{14}^{e}=\left(2 e_{c 0} / n_{c 0}\right)\left[-\sin v_{c n}(t)+\sin v_{c 0}\right] \\
& \delta \Phi_{15}^{e}=-\frac{e_{c 0}}{2 n_{c 0}}\left[-\cos \left(v_{c n}(t)-2 v_{c 0}\right)-4 \cos v_{c 0}+5 \cos v_{c n}(t)\right.\left.+3 n_{c 0} \tau \sin v_{c 0}\right] \\
& \delta \Phi_{21}^{e}=-\frac{1}{2} e_{c 0}\left[-8 \sin \left(v_{c n}(t)-2 v_{c 0}\right)+3 \sin \left(2 v_{c n}(t)-v_{c 0}\right)\right. \\
&\left.+12 n_{c 0} \tau \cos v_{c n}(t)+30 n_{c 0} \tau \cos v_{c 0}+5 \sin v_{c 0}-16 \sin v_{c n}(t)\right] \\
& \delta \Phi_{22}^{e}=e_{c 0}\left[-2 \cos \left(v_{c n}(t)-2 v_{c 0}\right)+\cos v_{c 0}+\cos v_{c n}(t)\right. \\
& \delta \Phi_{24}^{e}=-\frac{e_{c 0}}{2 n_{c 0}}\left[\cos \left(2 v_{c n}(t)-v_{c 0}\right)-5 \cos v_{c 0}+4 \cos v_{c n}(t)\right. \\
&\left.+6 n_{c 0} \tau \sin v_{c 0}\right] \\
& \delta \Phi_{25}^{e}=-\frac{e_{c 0}}{n_{c 0}}\left[-\sin \left(v_{c n}(t)-2 v_{c 0}\right)-\sin \left(2 v_{c n}(t)-v_{c 0}\right)\right. \\
&\left.+3 n_{c 0} \tau \cos v_{c n}(t)+3 n_{c 0} \tau \cos v_{c 0}+\sin v_{c 0}-\sin v_{c n}(t)\right]
\end{aligned}
$$




$$
\begin{gathered}
\delta \Phi_{33}^{e}=(1 / 2) e_{c 0}\left[2 \cos \left(v_{c n}(t)-v_{c 0}\right)+2 \cos v_{c n}(t)-\cos v_{c 0}\right] \\
\delta \Phi_{36}^{e}=-\frac{e_{c 0}}{2 n_{c 0}}\left[-\sin \left(v_{c n}(t)-2 v_{c 0}\right)-\sin \left(2 v_{c n}(t)-v_{c 0}\right)\right. \\
\left.-\sin v_{c 0}+\sin v_{c n}(t)\right] \\
\delta \Phi_{41}^{e}=-e_{c 0} n_{c 0}\left[-2 \sin \left(v_{c n}(t)-2 v_{c 0}\right)-3 \sin \left(2 v_{c n}(t)-v_{c 0}\right)\right. \\
\left.\quad+6 n_{c 0} \tau \cos v_{c n}(t)+3 \sin v_{c 0}-2 \sin v_{c n}(t)\right] \\
\delta \Phi_{42}^{e}=-e_{c 0} n_{c 0}\left[-\cos \left(v_{c n}(t)-2 v_{c 0}\right)+\cos v_{c n}(t)\right] \\
\delta \Phi_{44}^{e}=e_{c 0}\left[\cos \left(2 v_{c n}(t)-v_{c 0}\right)+\cos v_{c 0}-2 \cos v_{c n}(t)\right] \\
\delta \Phi_{45}^{e}=-(1 / 2) e_{c 0}\left[-\sin \left(v_{c n}(t)-2 v_{c 0}\right)-4 \sin \left(2 v_{c n}(t)-v_{c 0}\right)\right. \\
\left.\quad+6 n_{c 0} \tau \cos v_{c n}(t)+\sin v_{c 0}+4 \sin v_{c n}(t)\right] \\
\delta \Phi_{51}^{e}=-e_{c 0} n_{c 0}\left[-4 \cos \left(v_{c n}(t)-2 v_{c 0}\right)+3 \cos \left(2 v_{c n}(t)-v_{c 0}\right)\right. \\
\left.\quad+6 n_{c 0} \tau \sin v_{c n}(t)-9 \cos v_{c 0}-2 \cos v_{c n}(t)\right] \\
\delta \Phi_{52}^{e}=e_{c 0} n_{c 0}\left[2 \sin \left(v_{c n}(t)-2 v_{c 0}\right)+3 \sin v_{c 0}-\sin v_{c n}(t)\right] \\
\delta \Phi_{54}^{e}=e_{c 0}\left[-\sin \left(2 v_{c n}(t)-v_{c 0}\right)-\sin v_{c 0}+2 \sin v_{c n}(t)\right] \\
\delta \Phi_{55}^{e}=-e_{c 0}\left[\cos \left(v_{c n}(t)-2 v_{c 0}\right)-2 \cos \left(2 v_{c n}(t)-v_{c 0}\right)\right. \\
\left.\quad-3 n_{c 0} \tau \sin v_{c n}(t)-\cos v_{c 0}+2 \cos v_{c n}(t)\right] \\
\delta \Phi_{63}^{e}=e_{c 0} n_{c 0}\left[-\sin v_{c n}(t)+\sin v_{c 0}\right] \\
\delta \Phi_{66}^{e}=-(1 / 2) e_{c 0}\left[\cos \left(v_{c n}(t)-2 v_{c 0}\right)-2 \cos v_{c 0}+\cos v_{c n}(t)\right]
\end{gathered}
$$

\section{Appendix B. Coefficients of $\delta x(\psi)$}

Among the coefficients of $\delta \boldsymbol{x}(\psi)$ given in Eqs. (20)-(25), those that also appear in Eqs. (34)-(39) are written as follows:

$$
\begin{aligned}
A= & \frac{1}{2} \gamma\left(5 \cos ^{2} i_{c 0}-1\right) x_{n 0}, \quad B=\frac{1}{2 n_{c 0}} \gamma\left(5 \cos ^{2} i_{c 0}-1\right) \dot{x}_{n 0} \\
D= & \left(\gamma / n_{c 0}\right) \sin ^{2} i_{c 0} \sin \theta_{c 0}\left(n_{c 0} \sin \theta_{c 0} z_{n 0}+\cos \theta_{c 0} \dot{z}_{n 0}\right) \\
E= & \left(\gamma / n_{c 0}\right) \sin ^{2} i_{c 0} \cos \theta_{c 0}\left(n_{c 0} \sin \theta_{c 0} z_{n 0}+\cos \theta_{c 0} \dot{z}_{n 0}\right) \\
F= & 3\left[\gamma\left(2-3 \cos ^{2} i_{c 0}-3 \sin ^{2} i_{c 0} \cos ^{2} \theta_{c 0}\right)-3 e_{c 0} \cos v_{c 0}\right] x_{n 0} \\
& -3\left[2 \gamma \sin ^{2} i_{c 0} \sin \theta_{c 0}+3 e_{c 0} \sin v_{c 0}\right] y_{n 0}-8 \gamma \sin 2 i_{c 0} \sin \theta_{c 0} z_{n 0} \\
& -\left(1 / n_{c 0}\right)\left(3 e_{c 0} \sin v_{c 0} \dot{x}_{n 0}-2 \gamma \sin 2 i_{c 0} \cos \theta_{c 0} \dot{z}_{n 0}\right) \\
C_{1}^{x}= & (1 / 8)\left\{\gamma\left[2\left(19 \cos ^{2} i_{c 0}-21\right) \cos ^{2} \theta_{c 0}-43 \cos ^{2} i_{c 0}+29\right]\right. \\
& \left.-48 e_{c 0} \sin v_{c 0}\right\} x_{n 0}-(13 / 3) \gamma \sin 2 i_{c 0} z_{n 0} \\
& -(2 / 3)\left\{5 \gamma \sin ^{2} i_{c 0} \sin \theta_{c 0} \cos \theta_{c 0}+3 e_{c 0} \sin v_{c 0}\right\} y_{n 0} \\
& +\frac{1}{12 n_{c 0}}\left\{\gamma\left(\cos ^{2} i_{c 0}-7\right) \sin \theta_{c 0} \cos \theta_{c 0}-24 e_{c 0} \sin v_{c 0}\right\} \dot{x}_{n 0} \\
& -\frac{2}{3 n_{c 0}} \gamma \sin 2 i_{c 0} \cos \theta_{c 0} \dot{z}_{n 0} \\
S_{1}^{x}= & (3 / 8) \gamma\left(5 \cos ^{2} i_{c 0}-3\right) \sin 2 \theta_{c 0} x_{n 0}+(2 / 3) \gamma \sin ^{2} i_{c 0} \cos 2 \theta_{c 0} y_{n 0} \\
& +\frac{1}{3 n_{c 0}} \gamma \sin 2 i_{c 0}\left(n_{c 0} \cos \theta_{c 0} z_{n 0}+\sin \theta_{c 0} \dot{z}_{n 0}\right) \\
& +\frac{1}{24 n_{c 0}}\left[\gamma\left\{2\left(11 \cos i_{c 0}-5\right) \cos ^{2} \theta_{c 0}+25 \cos ^{2} i_{c 0}-7\right\}\right. \\
& \left.-48 e_{c 0} \cos v_{c 0}\right] \dot{x}_{n 0} \\
C_{1}^{y}= & (1 / 6)\left(23 \cos ^{2} i_{c 0}-17\right) \sin \theta_{c 0} \cos \theta_{c 0} x_{n 0} \\
& +\frac{1}{3}\left[\gamma\left(13 \sin ^{2} i_{c 0} \cos \theta_{c 0}^{2}+11 \cos i_{c 0}-8\right)-3 e_{c 0} \cos v_{c 0}\right] y_{n 0} \\
& -\frac{1}{4 n_{c 0}}\left\{\gamma\left[2\left(3 \cos ^{2} i_{c 0}-5\right) \cos \theta_{c 0}^{2}+9 \cos ^{2} i_{c 0}+1\right\}\right. \\
& \left.-8 e_{c 0} \cos v_{c 0}\right\} \dot{x}_{n 0}-\frac{2}{3 n_{c 0}} \gamma \sin 2 i_{c 0}\left(n_{c 0} \cos \theta_{c 0} z_{n 0}+\sin \theta_{c 0} \dot{z}_{n 0}\right) \\
& \\
&
\end{aligned}
$$

$$
\begin{aligned}
S_{1}^{y}= & \frac{1}{12} \gamma\left[2\left(73-79 \cos ^{2} i_{c 0}\right) \cos ^{2} \theta_{c 0}+5\left(23 \cos ^{2} i_{c 0}-17\right)\right] x_{n 0} \\
& +12 e_{c 0} \cos v_{c 0} x_{n 0}+\frac{1}{3}\left(11 \gamma \sin ^{2} i_{c 0} \sin 2 \theta_{c 0}-9 e_{c 0} \sin v_{c 0}\right) y_{n 0} \\
& +\frac{1}{6 n_{c 0}}\left[\gamma\left(29 \cos ^{2} i_{c 0}-11\right) \sin \theta_{c 0} \cos \theta_{c 0}+12 e_{c 0} \sin v_{c 0}\right] \dot{x}_{n 0} \\
& +\frac{2}{3 n_{c 0}} \gamma \sin 2 i_{c 0}\left(14 n_{c 0} \sin \theta_{c 0} z_{n 0}+2 \cos \theta_{c 0} \dot{z}_{n 0}\right) \\
C_{2}^{y}= & (1 / 6)\left(\gamma \sin ^{2} i_{c 0} \sin 2 \theta_{c 0}+3 e_{c 0} \sin v_{c 0}\right) x_{n 0} \\
& -(1 / 3) \gamma \sin ^{2} i_{c 0} \cos 2 \theta_{c 0} y_{n 0} \\
& +\frac{1}{2 n_{c 0}}\left\{\gamma\left(\cos ^{2} i_{c 0}-\sin { }^{2} i_{c 0} \cos ^{2} \theta_{c 0}\right)-e_{c 0} \cos v_{c 0}\right\} \dot{x}_{n 0} \\
& +\frac{1}{3 n_{c 0}} \gamma \sin 2 i_{c 0}\left(n_{c 0} \cos \theta_{c 0} z_{n 0}+\sin \theta_{c 0} \dot{z}_{n 0}\right) \\
C_{3}^{y}= & -\frac{1}{4 n_{c 0}} \gamma\left(1+\cos ^{2} i_{c 0}\right)\left(n \sin 2 \theta_{c 0} x_{n 0}+\cos 2 \theta_{c 0} \dot{x}_{n 0}\right) \\
C_{1}^{z}= & -\frac{1}{3 n_{c 0}} \gamma \sin 2 i_{c 0}\left(n_{c 0} \sin \theta_{c 0} x_{n 0}+2 \cos \theta_{c 0} \dot{x}_{n 0}\right) \\
& +(1 / 12)\left\{\gamma\left[\left(8 \sin ^{2} i_{c 0}-11\right) \cos ^{2} \theta_{c 0}-26 \cos ^{2} i_{c 0}+17\right]\right. \\
& \left.+12 e_{c 0} \cos v_{c 0}\right\} z_{n 0}-\frac{1}{12 n_{c 0}} \gamma\left(2 \cos i_{c 0}+1\right) \sin 2 \theta_{c 0} \dot{z}_{n 0} \\
S_{1}^{z}= & -\frac{1}{3 n_{c 0}} \gamma \sin 2 i_{c 0}\left(n_{c 0} \sin \theta_{c 0} x_{n 0}+\cos \theta_{c 0} \dot{x}_{n 0}\right) \\
+ & (1 / 12)\left\{\gamma\left[\left(4 \sin ^{2} i_{c 0}+5\right) \sin 2 \theta_{c 0}-12 e_{c 0} \sin v_{c 0}\right] z_{n 0}\right. \\
& +\frac{1}{12 n_{c 0}}\left\{\gamma\left[2\left(10 \cos ^{2} i_{c 0}-7\right)+19-46 \cos ^{2} i_{c 0}\right]\right. \\
& \left.-12 e \cos v_{c 0}\right\} \dot{z}_{n 0}
\end{aligned}
$$

\section{References}

1) Hill, G.: Researches in the Lunar Theory, American Journal of Mathematics, 1(1878), pp5-26.

2) Clohessy, W. H. and Wiltshire, R. S.: Terminal Guidance System for Spacecraft Rendezvous, J. Aerospace Sciences, 27(1960), pp. 653-658.

3) Tschauner, J. and Hempel, P.: Rendezvous zu Einemin in Elliptischer Bahn Umlaufenden Ziel, Acta Astronautica, 11(1965), pp. 104-109.

4) Melton, R. G.: Time-Explicit Representation of Relative Motion Between Elliptical Orbits, J. Guid. Control Dynam., 23(2000), pp. 604-610.

5) Chao, C. C.: Applied Orbit Perturbation and Maintenance, The Aerospace Press, California, 2005, pp. 7-16.

6) Montenbruck, O. and Gill, E.: Satellite Orbits, Springer, Berlin, 2000, pp. 54-60.

7) Yamada, K., Shima, T., Yoshikawa, S.: Effect of J2 Perturbations on Relative Spacecraft Position in Near-Circular Orbits, J. Guid. Control Dynam., 33(2010), pp. 584-590.

8) Yamada, K., Kimura, M., Shima, T., and Yoshikawa, S.: New State Transition Matrix for Formation Flying in J2-Perturbed Elliptic Orbits, J. Guid. Control Dynam., 35(2012), pp. 536-547.

9) D'Amico, S. and Montenbruck, O.: Proximity Operations of Formation-Flying Spacecraft Using an Eccentricity/Inclination Vector Separation, J. Guid. Control Dynam., 29(2006), pp. 554-563.

10) Montenbruck, O., Kirschner, M., D'Amico, S., and Bettadpur, S. E/I vector separation for safe switching of the GRACE formation, ScienceDirect, Aerospace Science and Technology, 10(2006), pp. $628-635$.

11) D'Amico, S.: Autonomous Formation Flying in Low Earth Orbit, Ph.D Thesis, Technical University of Delft, 2010. 